Georgian Mathematical Journal

$\mathbf{1}(1994)$, No. $1,47-52$

\title{
MEASURES OF CONTROLLABILITY
}

\author{
J. L. LIONS
}

\begin{abstract}
We introduce here a new notion, the measure of controllability aimed at expressing that one system is "more controllable" than another one. First estimates are given.
\end{abstract}

\section{INTRODUCTION}

Let $\Omega$ be an open set in $\mathbb{R}^{n}$, bounded or not, with boundary $\Gamma$, smooth or not.

In the domain $\Omega$ and for $t>0$, we consider the system whose state $y: y(x, t)=y(x, t ; v)$ is given as follows:

$$
\frac{\partial y}{\partial t}+A y=v(x, t) \chi_{\mathcal{O}} \quad \text { in } \Omega \times\{t>0\},
$$

where $A=$ second order elliptic operator in $\Omega$ (its coefficients are not necessarily smooth and they may depend on $t$ ),

$\mathcal{O}=$ open set $\subset \Omega$,

$\chi_{\mathcal{O}}=$ characteristic function of $\mathcal{O}$,

$v=v(x, t)=$ control function.

We add to (1.1) the initial and boundary conditions respectively given by

$$
y(x, 0)=y^{0}(x) \text { in } \Omega, \quad y^{0} \text { given in } L^{2}(\Omega),
$$

and

$$
y=0 \quad \text { on } \Gamma \times\{t>0\} .
$$

Under reasonable conditions on the coefficients of $A$ (cf.for instance J.L.Lions $[3]$ ), and assuming that

$$
v \in L^{2}(\mathcal{O} \times(0, T)),
$$

1991 Mathematics Subject Classification. 49J20. 
equations (1.1),(1.2),(1.3) admit a unique solution $y$, which is such that

$$
y, \frac{\partial y}{\partial x_{i}} \in L^{2}(\Omega \times(0, T)) .
$$

This defines the state of the system, with distributed control with support in $\mathcal{O}$.

Remark 1.1. Boundary condition (1.3) is taken here to fix ideas. What follows readily applies to other situations corresponding to other boundary conditions.

Remark 1.2. All what follows readily extends to higher order parabolic equations, to systems of parabolic equations and actually to all evolution equations, provided they are linear. This will be reported elsewhere. Cf. also the Remarks of the last section of this paper.

Remark 1.3. One knows that (J.L.Lions [3]) after a possible change on a set of 0 measure, the function $t \rightarrow y(t)=y(\cdot, t)$ is continuous from $[0, T] \rightarrow$ $L^{2}(\Omega)$.

Approximate controllability is defined as follows (cf. for instance J.L.Lions [4]). We are given $T$ and $y^{1} \in L^{2}(\Omega)$. Let $B$ denote the unit ball in $L^{2}(\Omega)$ and let $\beta$ be a positive number arbitrarily small.

It is known (J.L.Lions [5]) that, when $v$ spans $L^{2}(\mathcal{O} \times(0, T))$, the functions $y(\cdot, T ; v)$ describe an affine space in $L^{2}(\Omega)$ which is dense in $L^{2}(\Omega)$. Therefore one can always find functions $v$ (controls) such that

$$
y(T ; v) \in y^{1}+\beta B
$$

and there are infinitely many $v$ 's such that (1.6) takes place. One says that the system is approximately controllable. It is natural to look for the (actually unique) element $v$ such that

$$
\frac{1}{2} \iint_{\mathcal{O} \times(0, T)} v^{2} d x d t=\min
$$

where $v$ is restricted to those elements such that (1.6) takes place.

The question we want to address here is the following: when can we say that a system is more controllable than another one?

In this question we assume that $\Omega$ and that $\mathcal{O}$ do not change. Then the min in (1.7) is a quantity which depends on $A, y^{0}, y^{1}$ and $\beta$ and $T$. We write

$$
\begin{aligned}
& \inf _{v} \frac{1}{2} \iint_{\mathcal{O} \times(0, T)} v^{2} d x d t=M\left(A, y^{0}, y^{1}, \beta, T\right), \\
& y(T ; v) \in y^{1}+\beta B .
\end{aligned}
$$


We have to introduce a quantity which is independent of $y^{0}$ and of $y^{1}$ but which only depends on the sets described by $y^{0}$ and by $y^{1}$.

We shall assume

$$
y^{0} \in \alpha_{0} B, \quad y^{1} \in \alpha_{1} B
$$

and we introduce as a "measure of controllability" the quantity

$$
M\left(A, \alpha_{0}, \alpha_{1}, \beta, T\right)=\sup _{\substack{y^{0} \in \alpha_{0} B \\ y^{1} \in \alpha_{1} B}} M\left(A, y^{0}, y^{1}, \beta, T\right) .
$$

Remark 1.4. This quantity seems to be introduced here for the first time. The study of the function

$$
A \rightarrow M\left(A, \alpha_{0}, \alpha_{1}, \beta, T\right)
$$

leads to many seemingly interesting open questions. We shall return to these questions in other occasions.

Remark 1.5. It is not obvious that the quantity introduced in (1.9) is always finite. Indeed this quantity is finite iff $\beta>\alpha_{1}$.

Remark 1.6. We shall give below a number of simple formulas reducing the number of variables $\alpha_{0}, \alpha_{1}, \beta$ to actually one variable.

We are now going to give a formula for $M\left(A, \alpha_{0}, \alpha_{1}, \beta, T\right)$ which is based on duality arguments.

\section{Duality Formula for the Measure of Controllability}

We introduce the decomposition

$$
y(x, t ; v)=y(v)=y_{0}+z(v)
$$

where

$$
\begin{aligned}
& \frac{\partial y_{0}}{\partial t}+A y_{0}=0, \\
& y_{0}(0)=y^{0}, \quad y_{0}=0 \quad \text { on } \quad \Gamma \times(0, T)
\end{aligned}
$$

and

$$
\begin{aligned}
& \frac{\partial z}{\partial t}+A z=v \chi_{\mathcal{O}}, \\
& z(0)=0, \quad z=0 \quad \text { on } \Gamma \times(0, T) .
\end{aligned}
$$

Then

$$
\begin{aligned}
& M\left(A, y^{0}, y^{1}, \beta, T\right)=\inf \frac{1}{2} \iint_{\mathcal{O} \times(0, T)} v^{2} d x d t \\
& z(T ; v) \in y^{1}-y_{0}(T)+\beta B
\end{aligned}
$$


We introduce the convex functions defined by

$$
\begin{gathered}
F_{0}(v)=\frac{1}{2} \iint_{\mathcal{O} \times(0, T)} v^{2} d x d t, \quad v \in L^{2}(\mathcal{O} \times(0, T)), \\
F_{1}(f)=\left\{\begin{array}{l}
0 \text { if } f \in y^{1}-y_{0}(T)+\beta B, \\
+\infty \text { otherwise in } L^{2}(\Omega) .
\end{array}\right.
\end{gathered}
$$

We define the linear operator $L$ by

$$
L v=z(T ; v) .
$$

One has

$$
L \in \mathcal{L}\left(L^{2}(\mathcal{O} \times(0, T)) ; L^{2}(\Omega)\right) .
$$

With those notations (this is only a matter of definition)

$$
M\left(A, y^{0}, y^{1}, \beta, T\right)=\inf _{v \in L^{2}(\mathcal{O} \times(0, T))} F_{0}(v)+F_{1}(L v) .
$$

The next step is to use Fenchel-Rockafellar duality (cf. T.R.Rockafellar [6] and the presentation made in I.Ekeland and R.Temam [1]).

In general, the conjugate function $F_{i}^{*}$ of $F_{i}$ is defined by

$$
F_{i}^{*}(f)=\sup _{\widehat{f}}\left[(f, \widehat{f})-F_{i}(\widehat{f})\right]
$$

With these definitions, one has

$$
\begin{aligned}
& F_{0}^{*}(v)=F_{0}(v), \\
& F_{1}^{*}(f)=\left(f, y^{1}-y_{0}(T)\right)+\beta\|f\|, \\
& \text { where }\|f\| \in\left(\int_{\Omega} f^{2} d x\right)^{\frac{1}{2}} .
\end{aligned}
$$

Let $L^{*}$ denote the adjoint of $L$. Then (T.R.Rockafellar, loc.cit.)

$$
\begin{aligned}
& \inf _{v \in L^{2}(\mathcal{O} \times(0, T))} F_{0}(v)+F_{1}(L v)= \\
& -\inf _{f \in L^{2}(\Omega)} F_{0}^{*}\left(L^{*} f\right)+F_{1}^{*}(-f) .
\end{aligned}
$$

The operator $L^{*}$ is given as follows. If $f$ is given in $L^{2}(\Omega)$, we solve

$$
\begin{aligned}
& -\frac{\partial \psi}{\partial t}+A^{*} \psi=0, \quad t<T, \\
& \psi(x, T)=f(x) \quad \text { in } \quad \Omega, \\
& \psi=0 \text { on } \Gamma \times\{t<T\}
\end{aligned}
$$

where $A^{*}=$ adjoint of $A$.

This problem admits a unique solution $\psi(x, t)=\psi(x, t ; f)=\psi(f)$. 
Then one easily verifies that

$$
L^{*} f=\psi \chi_{\mathcal{O}} .
$$

Using this result, (2.11), and (2.10), we obtain

$$
\begin{aligned}
M\left(A, y^{0}, y^{1}, \beta, T\right)= & -\inf _{f \in L^{2}(\Omega)} \frac{1}{2} \iint_{\mathcal{O} \times(0, T)} \psi^{2} d x d t- \\
& -\left(f, y^{1}-y_{0}(T)\right)+\beta\|f\| .
\end{aligned}
$$

If we multiply (2.12) by $y_{0}$, we obtain after integration by parts

$$
-\left(f, y_{0}(T)\right)+\left(\psi(0), y^{0}\right)=0
$$

so that (2.14) can be written

$$
\begin{aligned}
& M\left(A, y^{0}, y^{1}, \beta, T\right)= \\
& =-\inf _{f \in L^{2}(\Omega)} \frac{1}{2} \iint_{\mathcal{O} \times(0, T)} v^{2} d x d t- \\
& -\left(f, y^{1}\right)+\left(\psi(0), y^{0}\right)+\|f\| . \quad \square
\end{aligned}
$$

By definition

$$
\begin{aligned}
& M\left(A, \alpha_{0}, \alpha_{1}, \beta, T\right)= \\
& =\sup _{y^{0} \in \alpha_{0} B, y^{1} \in \alpha_{1} B} M\left(A, y^{0}, y^{1}, \beta, T\right)=(\text { using }(2.16))= \\
& =-\inf _{y^{0} \in \alpha_{0} B, y^{1} \in \alpha_{1} B, f \in L^{2}(\Omega)} \frac{1}{2} \iint_{\mathcal{O} \times(0, T)} \psi^{2} d x d t- \\
& -\left(f, y^{1}\right)+\left(\psi(0), y^{0}\right)+\beta\|f\|,
\end{aligned}
$$

i.e.

$$
\begin{aligned}
& M\left(A, \alpha_{0}, \alpha_{1}, \beta, T\right)= \\
& =\inf _{f}\left[\frac{1}{2} \iint_{\mathcal{O} \times(0, T)} \psi^{2} d x d t+\right. \\
& \left.+\left(\beta-\alpha_{1}\right)\|f\|-\alpha_{0}\|\psi(0)\|\right] .
\end{aligned}
$$

In summary:

the measure of controllability is given by formula (2.18), where $\psi=\psi(f)$ is given by (2.12).

Remark 2.1. One can show that the $\inf _{f}$ in (2.18) is finite iff $\beta>\alpha_{1}$. 
One has

$$
M\left(A, \alpha_{0}, \alpha_{1}, \beta, T\right)=M\left(A, \alpha_{0}, 0, \beta-\alpha_{1}, T\right), \quad \beta>\alpha_{1} .
$$

Therefore it suffices to consider the following situation:

$$
\begin{aligned}
& \sup _{y^{0} \in \alpha \beta} \inf \frac{1}{2} \iint_{\mathcal{O} \times(0, T)} v^{2} d x d t=M_{0}(A, \alpha, \beta, T), \\
& y(T ; v) \in \beta B
\end{aligned}
$$

(Then $\left.M\left(A, \alpha_{0}, \alpha_{1}, \beta, T\right)=M_{0}\left(A, \alpha_{0}, \beta-\alpha_{1}, T\right)\right)$.

One verifies directly that

$$
\begin{gathered}
M_{0}(A, \alpha, \beta, T)=\alpha^{2} M_{0}\left(A, 1, \frac{\beta}{\alpha}, T\right) \\
M_{0}(A, \alpha, \beta, T)= \begin{cases}0 & \text { for } \beta \text { large enough, } \\
\text { increases to }+\infty \text { as } \beta \text { decreases to } 0 .\end{cases}
\end{gathered}
$$

Remark 2.2. Formula (2.18) is constructive. One can deduce from it numerical algorithms for the approximation of $M$. Cf. R.Glowinski and J.L.Lions [2].

\section{REFERENCES}

1. I.Ekeland and R.Temam, Analyse Convexe et problèmes variationnels. Dunod, Gauthier Villars, Paris-Bruxeles-Montréal, 1974.

2. R.Glowinski and J.L.Lions, To appear in Acta Numerica, 1993.

3. J.L.Lions, Equations Differentielles Opérationnelles et Problèmes aux limites. Springer-Verlag, Berlin-New York, 1961.

4. J.L.Lions, Exact Controllability for distributed systems. Some trends and some problems. Applied and Industrial Mathematics, R.Spigler (ed.), 59-84, Kluwer, 1991.

5. —_ Controle Optimal de systèmes gouvernés par les équations aux dérivées partielles. Dunod, Gauthier-Villars, Paris, 1968.

6. T.R.Rockafellar, Duality and stability in extremum problems involving convex functions. Pac. J. Math. 21(1967), 167-187.

(Received 26.11.1992)

Author's address:

Collège de France, 3 rue d'Ulm

F-75231 Paris Cedex 05, France 